\title{
Unusual Histology of Eosinophilic Myenteric Ganglionitis: A Case Report
}

\author{
Hyekyung Lee · Dongwook Kang \\ Heejin $\mathrm{Kim}^{1} \cdot$ Byungsun $\mathrm{Cho}^{2}$ \\ Jeho Jang ${ }^{2}$ \\ Departments of Pathology, ${ }^{1}$ Radiology, and \\ ${ }^{2}$ Surgery, Eulji University School of Medicine, \\ Daejeon, Korea
}

Received: June 2, 2016

Revised: August 5, 2016

Accepted: September 7, 2016

\section{Corresponding Author}

Jeho Jang, MD

Department of Surgery, Eulji University School of

Medicine, 77 Gyeryong-ro 771 beon-gil, Jung-gu,

Daejeon 34824, Korea

Tel: $+82-42-259-1272$

Fax: +82-42-259-1111

E-mail: jhjang@eulji.ac.kr
Eosinophilic myenteric ganglionitis is a disorder characterized by infiltration of the Auerbach myenteric plexus by eosinophils. As a cause of chronic intestinal pseudo-obstruction (CIPO), eosinophilic myenteric ganglionitis has been rarely reported and the majority of the reported cases in the literature were children. We experienced a case of eosinophilic myenteric ganglionitis associated with CIPO in a 53-year-old female patient. Histologic examination of the resected descending colon showed moderate eosinophilic infiltrates with hypogangliosis in the myenteric plexus. Immunohistochemical study revealed increased number of CD4-positive lymphocytes and stronger but scantier glial fibillary acid protein expression in the inflamed myenteric plexus.

Key Words: Eosinophils; Ganglionitis; Pseudo-obstruction
Chronic intestinal pseudo-obstruction (CIPO) represents a rare and highly morbid syndrome characterized by impaired gastrointestinal propulsion together with symptoms of bowel obstruction in the absence of any lesions occluding the gut lumen. ${ }^{1,2}$ The diagnosis of CIPO is mainly clinical and confirmed by endoscopic or radiologic findings. Chronic intestinal pseudoobstruction has many underlying causes and eosinophilic myenteric ganglionitis has rarely been reported as a cause of CIPO. Eosinophilic myenteric ganglionitis is known as a neuropathy which develops mainly in children and shows no degenerative pathologic findings such as neuronal loss, and no lymphocytic infiltrates. ${ }^{3,4}$ We present a middle aged female patient with eosinophilic myenteric ganglionitis as the underlying cause of CIPO. In our case, we observed hypogangliosis with moderate eosinophilic infiltrates admixed with CD4-positive T cells, which has not been described before.

\section{CASE REPORT}

A 53-year-old woman presented to the emergency department with a 2-week history of abdominal distention and constipation. She had a previous history of having undergone a myomectomy
15 years ago and has been under thyroid hormone replacement therapy for 10 years. She suffered severe constipation for a long time that was refractory to stool softeners. Seven years ago, she had visited our hospital due to the same symptoms. At the time, abdominopelvic computed tomography revealed markedly dilated entire colon with feces and tapered narrowing in the distal portion (Fig. 1A, B). Colonoscopic mucosal biopsy showed mild lymphoplasmacytic infiltrates in the mucosa without muscularis mucosa component. At this visit, laboratory findings were within normal limits except for a slightly decreased TSH level (0.48 $\mathrm{IIU} / \mathrm{mL}$; normal range, 0.55 to 4.2). Serologic study of anti-Hu antibody, anti-Ri antibody, anti-Yo antibody, antineuromyelitis optica antibody, anti-antinuclear antibody, antithyroid-stimulating hormone receptor antibody, anti-thyroglobulin antibody, and anti-thyroid microsomal antibody were all negative. On abdominopelvic computed tomography, the entire colon was severely dilated and there was no significant difference between the previous and current images. Neither mural thickening nor any mass lesion was noted at the narrowed area.

The differential diagnoses included colonic obstruction due to stricture from inflammatory bowel disease or ischemic colitis and a mild form of congenital megacolon. Colonoscopic evalua- 

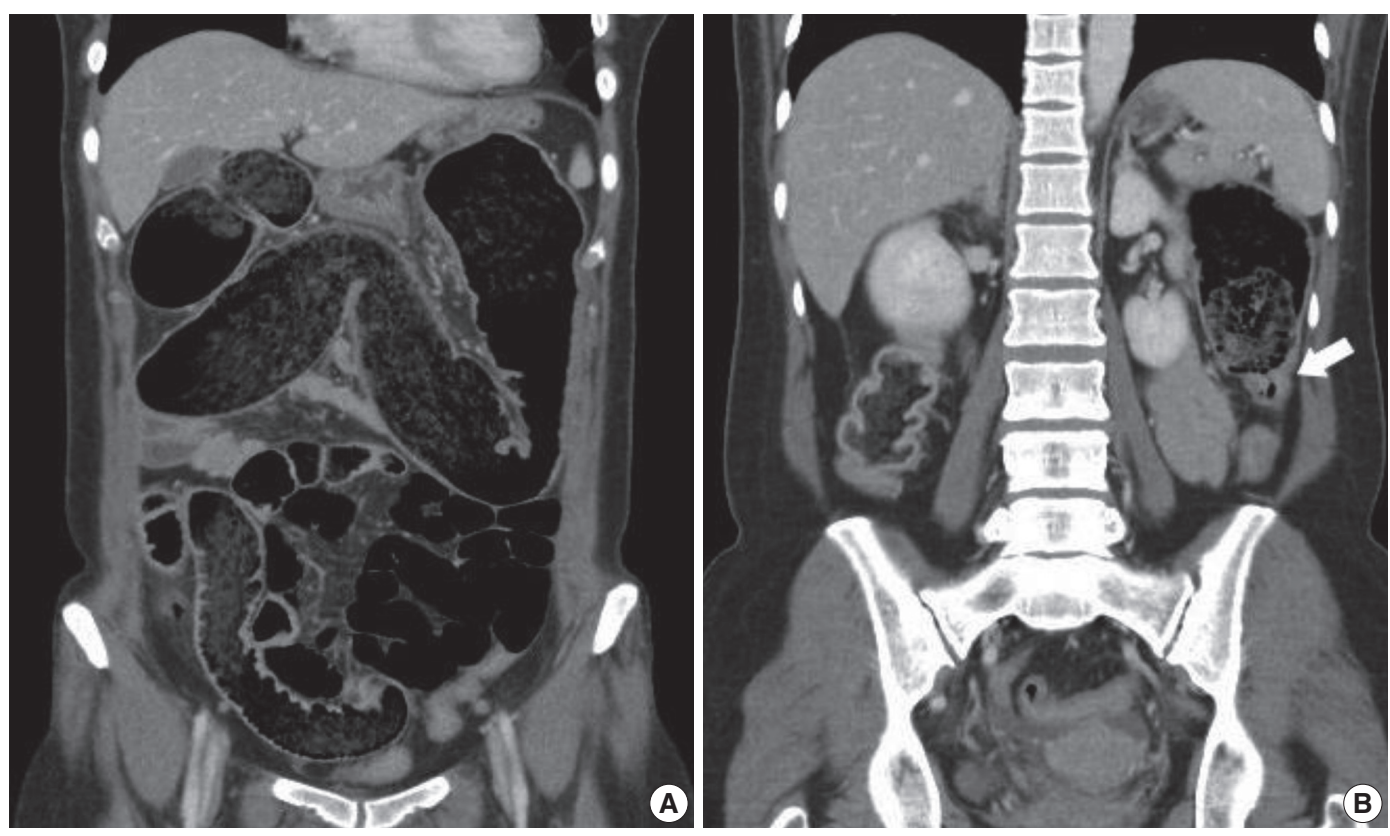

Fig. 1. Abdominopelvic computed tomography shows markedly dilated colon filled with feces (A). Transition point between the dilated proximal and collapsed distal colon is noted (B, arrow).

tion showed luminal narrowing in the descending colon without definite luminal obstructive lesions. Proximal advancement of the scope was not possible due to much fecal material and the patient's pain. After 3 days of conservative management with slow bowel preparation with polyethylene glycol solution, the distended abdomen was decompressed and the symptoms subsided. Colon transit time study showed a slow transit constipation pattern. Given concern for the possibility of intractable benign luminal stenosis due to adhesive structure or ischemic enteritis, segmental colectomy was performed laparoscopically. There was no evidence of adhesion or mechanical obstruction except for focal physiologic attachment between the visceral and parietal peritoneum. The patient's postoperative course was uncomplicated and the patient's symptoms resolved after the surgery.

\section{Pathologic features}

A segment of descending colon measuring $12 \mathrm{~cm}$ in length was received. Approximately half of the colonic segment was dilated (Fig. 2). The wall thickness appeared even without regional differences between the dilated and the narrowed portions. Except for the luminal dilatation, the colon was grossly unremarkable. Histologic examination revealed mild lymphoplasmacytic infiltrates with scattered eosinophils in the mucosa. The average number of eosinophils was less than 4 per high power field (HPF). The muscularis mucosa was thickened up to $660 \mu \mathrm{m}$ in the dilated area in contrast to the thin muscularis

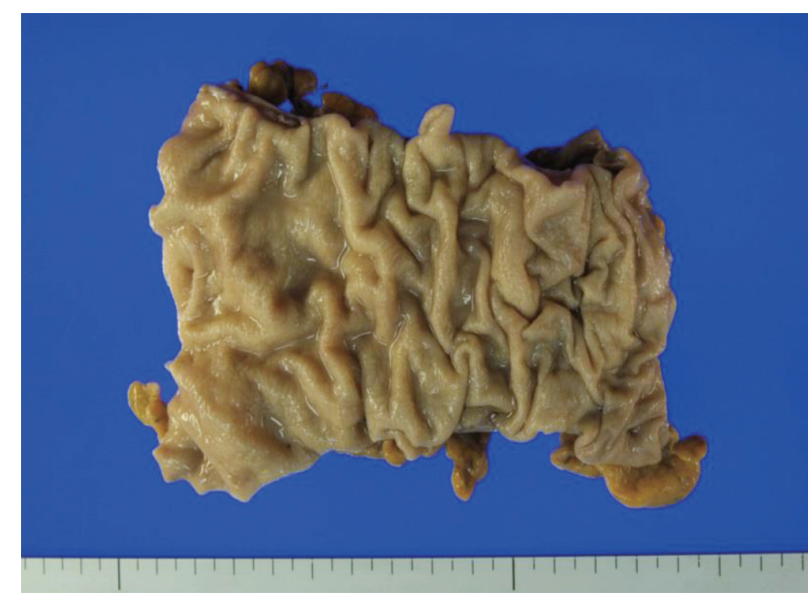

Fig. 2. Approximately half of the resected segment of the descending colon was dilated.

mucosa in the narrow area (Fig. 3A). Scattered mast cells were noted in the submucosa without significant eosinophilic infiltrates. The Meissner's plexuses were unremarkable. Intermuscular Auerbach's myenteric plexuses showed moderate eosinophilic infiltrates up to 22 per HPF (Fig. 3B) and hypogangliosis with atrophic reduction of the volume of nerve bundle, which became more evident with synaptophysin immunostain (Fig. 3C). The atrophic myenteric plexus was accompanied by increased fibrosis and admixed with some CD3 and CD4 dual positive lymphocytes (Fig. 3D) and a few CD8-positive lymphocytes on immunostain. Immunohistochemical study showed preserved positivity 

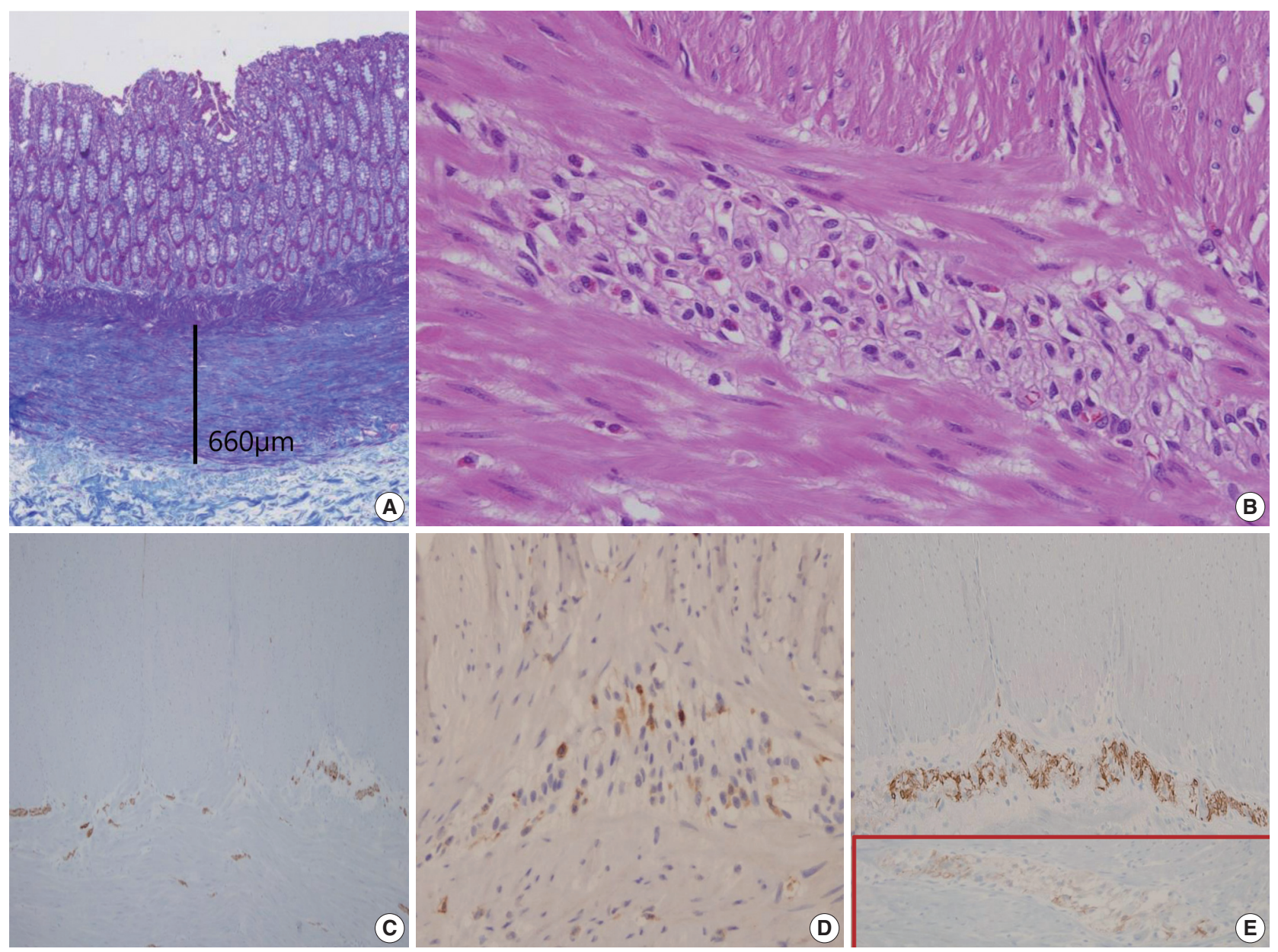

Fig. 3. Summary of histologic findings: thickened muscularis mucosa in the dilated colon (A), many eosinophils in Auerbach's myenteric plexus (B), hypogangliosis in synaptophysin immunostain (C), scattered CD4-positive lymphocytes in the affected myenteric plexus (D) and stonger but scantier glial fibrillary acidic protein positivity in our patient (E) in contrast to the control patient (in box).

of c-Kit and stronger but scantier glial fibrillary acidic protein (GFAP) reaction in the myenteric plexus when we compared the immunostain results with a comparable descending colon of a 60 year old female patient who received Hartmann's operation due to cancer (Fig. 3E). Bcl-2 stain for anti-apoptosis showed no definite difference between the two colons. There was no significant difference in the severity of the eosinophilic infiltrates in the myenteric plexus between the grossly dilated and narrow portions. However, there was regional difference in the eosinophilic infiltrates between the inner portion and the outer portion of the myenteric plexus. There was no eosinophilic infiltrates in the submucosa and the inner muscle proper in contrast to the increased number of eosinophils, lymphocytes, and histiocytes in the outer muscle layer, subserosa, and serosa. There was no evidence of peripheral blood eosinophilia. Based on these findings, a diagnosis of eosinophilic myenteric ganglionitis was made.

\section{DISCUSSION}

Digestive motility, an important pathogenic factor of CIPO, is a highly coordinated process and depends on smooth muscle contractility and the related pacemaker activity evoked by the interstitial cells of Cajal (ICCs). Both of these mechanisms are finely tuned by the intrinsic enteric nervous system (ENS) and the extrinsic sympathetic and parasympathetic nerves, independently from the central and peripheral nervous systems. Based on histology, CIPO can be classified into three major entities depending on the predominant involvement of enteric neurons, ICCs, or smooth muscle cells: neuropathies, mesenchymopathies, and myopathies.

Neuropathy can be classified into two major forms: (1) inflammatory neuropathies in which a significant inflammatory response is identified within nerve tissue and (2) degenerative neuropathies characterized by evidence of neurodegenerative aspects in 
the absence of an identifiable inflammatory response. Inflammatory neuropathy is also subdivided into lymphocytic and eosinophilic depending on the prominent infiltrating cells. Lymphocytic neuropathy is the main inflammatory neuropathy and eosinophilic neuropathy has rarely been reported. ${ }^{5}$ Distinctive findings of eosinophilic neuropathy has mainly been reported in children showing no degenerative pathologic findings such as neuronal loss and no lymphocytic infiltrates. We report a case of eosinophilic myenteric ganglionitis which developed in a middleaged female patient with the pathologic features of hypogangliosis and CD4-positive lymphocytic infiltrates. The pathogenesis of eosinophilic myenteric ganglionitis has not been clearly elucidated. However, interleukin 5 (IL-5), a well-known eosinophilic chemotactic factor, has been mentioned in eosinophilic myenteric ganglionitis. ${ }^{3}$ Although we did not evaluate IL-5, the increased number of $\mathrm{CD} 3$ and $\mathrm{CD} 4$ dual positive cells may suggest the increased production of IL-5 from the infiltrating CD4positive helper $\mathrm{T}$ cells for eosinophilic chemotaxis. A few scattered CD8-positive lymphocytes may be involved in the cytotoxic reaction of the nerve plexus resulting in hypogangliosis. Atrophic plexus and cell loss in neuroinflammatory disease were mentioned as the result of the activation of a caspase dependent mechanism; ${ }^{6}$ however, immunoreactivity of $\mathrm{Bcl}-2$ in our case showed no definite reduced positivity. The expression of GFAP in the myenteric plexus was an impressive finding. The myenteric plexus is composed of enteric neurons and enteric glial cells. Enteric glial cells are the main constituent of the ENS and the majority of the enteric glial cells are located in the myenteric plexus. Recently, the role of enteric glial cells as a neuroprotector and as an immune-modulator in inflammatory processes has been investigated ${ }^{7}$ and the myenteric GFAP-expressing glial subpopulation was reported to be susceptible to systemic inflammation. ${ }^{8}$ In our case, the stronger but scantier expression of GFAP seems to be related with the overproduction of GFAP by activated enteric glial cells for neuroprotection as a damaged myenteric plexus. The role of the myenteric plexus as an immunemodulator and barrier was evident in the findings of scarce inflammatory reaction in the inner muscle layer in contrast to mild to moderate inflammatory reaction in the outer muscle layer and the subserosa. This regional difference suggests the barrier function of the myenteric plexus to diffusible and systemic immune reactants supplied by the mesenteric bloods through the subserosal and outer muscular layer vessels. There being no difference in the eosinophilic infiltrates in the myenteric plexus between the dilated and narrow areas was also meaningful. Similarly, no difference in ENS abnormality between the affected and the nonaffected areas in Crohn disease has been described. ${ }^{9}$ This finding suggests the eosinophilic reaction as a systemic reaction, and the possible discrepancy between the pathologic findings and functional impairment. The pathologic findings such as eosinophilic infiltrates or neuronal loss can be the underlying causes of CIPO; however, the clinical functional impairment is more complex. Physiologic abnormality such as disturbed activity of neurotransmitters can be another factor to take into consideration. The increased number of mast cells in the submucosa may be the finding of the crosstalk between mast cells and eosinophils. ${ }^{10}$ The thickened muscularis mucosa in the dilated colon can be a compensatory response to prolonged high luminal pressure. The therapeutic effect of surgery is limited since CIPO often involves the entire gastrointestinal tract with a progressive nature. As surgical approach can aggravate the underlying condition, it should be reserved for only carefully selected patients. If a confirmed diagnosis of ganglionitis is made through a full-thickness biopsy of the intestinal wall, the patient might benefit from an anti-inflammatory or immunosuppressive therapy before the surgical procedure. However, in the absence of a transmural tissue diagnosis, patients with enteric ganglionitis cannot be subject to these nonoperative treatment options.

In conclusion, we think this colonic lesion might have begun as a systemic immune reaction showing eosinophilic myenteric ganglionitis and progressed into a chronic inflammatory lesion showing atrophic myenteric plexus, reduced ganglion cells, thickened muscularis mucosa, and subserosal fibrosis. These abnormal findings can be the basis of the clinical symptoms of CIPO. The diagnosis of CIPO is mainly clinical, and the histopathology of CIPO has frequently been reported as a frustrating experience by pathologists due to limited experience. Further combined clinical and histopathological studies are needed in order to enrich the understanding and management CIPO.

\section{Conflicts of Interest}

No potential conflict of interest relevant to this article was reported.

\section{REFERENCES}

1. De Giorgio R, Sarnelli G, Corinaldesi R, Stanghellini V. Advances in our understanding of the pathology of chronic intestinal pseudo-obstruction. Gut 2004; 53: 1549-52.

2. Lee BH, Kim N, Kang SB, et al. Two cases of chronic idiopathic intestinal pseudo-obstruction with different clinical features. J Neu- 
rogastroenterol Motil 2010; 16: 83-9.

3. Schäppi MG, Smith VV, Milla PJ, Lindley KJ. Eosinophilic myenteric ganglionitis is associated with functional intestinal obstruction. Gut 2003; 52: 752-5.

4. Chander B, Fiedler P, Jain D. Eosinophilic myenteric ganglionitis: a case of intestinal pseudo-obstruction in a 93-year-old female. J Clin Gastroenterol 2011; 45: 314-6.

5. De Giorgio R, Camilleri M. Human enteric neuropathies: morphology and molecular pathology. Neurogastroenterol Motil 2004; 16: 515-31.

6. De Giorgio R, Bovara M, Barbara G, et al. Anti-HuD-induced neuronal apoptosis underlying paraneoplastic gut dysmotility. Gastro- enterology 2003; 125: 70-9.

7. Lakhan SE, Kirchgessner A. Neuroinflammation in inflammatory bowel disease. J Neuroinflammation 2010; 7: 37.

8. Rosenbaum C, Schick MA, Wollborn J, et al. Activation of myenteric glia during acute inflammation in vitro and in vivo. PLoS One 2016; 11: e0151335.

9. De Giorgio R, Guerrini S, Barbara G, et al. Inflammatory neuropathies of the enteric nervous system. Gastroenterology 2004; 126: 1872-83.

10. De Winter BY, van den Wijngaard RM, de Jonge WJ. Intestinal mast cells in gut inflammation and motility disturbances. Biochim Biophys Acta 2012; 1822: 66-73. 